\title{
Existence and stability of steady-state solutions of the shallow-ice-sheet equation by an energy-minimization approach
}

\author{
Guillaume JOUVET, ${ }^{1}$ Jacques RAPPAZ, ${ }^{1}$ Ed BUELER, ${ }^{2}$ Heinz BLATTER ${ }^{3}$
}

\author{
${ }^{1}$ Mathematics Institute of Computational Science and Engineering, EPFL, CH-1015 Lausanne, Switzerland \\ ${ }^{2}$ Department of Mathematics and Statistics, University of Alaska Fairbanks, Fairbanks, Alaska 99775-6660, USA \\ ${ }^{3}$ Institute for Atmospheric and Climate Science, ETH Zürich, Universitätstrasse 16, CH-8092 Zürich, Switzerland \\ E-mail: blatter@env.ethz.ch
}

\begin{abstract}
The existence of solutions of the non-sliding shallow-ice-sheet equation on a flat horizontal bed with a mass balance linearly depending on altitude is proven for fixed margins. Free-margin solutions for the same mass balance do not exist. Fixed-margin solutions show unbounded shear stress and nonzero mass flux at the margin. Steady-state solutions with realistic margins, vanishing ice flux and vanishing shear stress are found numerically for ice sheets with Weertman-type sliding.
\end{abstract}

\section{INTRODUCTION}

Mathematical models for realistic ice sheets are generally unsolvable by analytical methods due to the nonlinearity of the equations, the complex geometry of the domain and the boundary conditions. Exact solutions of the shallow-icesheet equation are one method of analysing properties of the shallow-ice approximation (SIA), which is known to exhibit reduced regularity (singular derivatives) around ice-sheet summits and margins, where conditions for shallowness are violated. Indeed, the need for verification of numerical models has motivated the development of exact solutions for a variety of simplified sets of geometries, physical conditions, approximations and boundary conditions.

In this paper, we investigate two features of the shallowice-sheet equation for flat beds in a case for which no exact solutions are known. The first concerns the existence and stability of solutions for mass balance linearly dependent on altitude (case 1 in 'Numerical results' section). The second concerns the marginal singularity, where vertical surface inclination and vanishing ice thickness may result in a nonvanishing or even unbounded basal shear stress (case 2).

Exact solutions of ice-sheet equations with different degrees of sophistication have been studied. Vialov (1958) presented an isothermal steady state for constant accumulation. More realistic accumulation functions (Bueler, 2003) and time-dependent solutions, both isothermal and thermocoupled, were proposed by Halfar $(1981,1983)$ and Bueler and others $(2005,2007)$. These solutions use the fact that the equation can be separated by variables if the accumulation is a function of the horizontal coordinate only, or if the accumulation function has a specific dependence on elevation and time, and when the resulting integrals can be solved analytically (Greve and Blatter, 2009). If the accumulation is a more general function of the ice surface elevation, the equation is not straightforwardly separable and its solution requires at least a numerical ODE (ordinary differential equation) solver. Such quadrature can sometimes be considered 'exact' for verification purposes.

Solutions for mass-balance functions which increase with surface elevation have been studied to address the stability of ice sheets in a changing climate (Weertman, 1961). Bodvarsson (1955) addresses this question by constructing an exact solution for a plug flow driven purely by basal sliding, but neglecting any ice deformation. Numerical studies using such mass-balance functions are well known in the literature (e.g. Oerlemans, 1981; Van den Berg and others, 2006), but these studies fail to resolve the mathematical question of the existence of a solution of the continuum problem. Furthermore, all numerical studies of this question must accept large margin-approximation errors because of the singularity of the surface gradient at the margin (Bueler and others, 2005).

Although an unlimited mass balance growing linearly with elevation is not particularly realistic, we analyse the problem in some detail to learn about the mathematical qualities of the problem. This paper aims to give a mathematical proof of the existence of solutions to this shallow-ice-sheet model. In our approach, the solution is a minimizer of an energy functional. The novelty of this result (cf. Calvo and others, 2002; Antontsev and de Oliveira, 2008) is that the source term (the mass balance) in the stationary shallow-ice-sheet equation depends on the unknown ice thickness.

The existence is proven only for fixed ice-sheet margins. Steady-state solutions are also obtained by numerical solution of the transient equation using the time-steps of the temporal evolution as a fixed-point iteration. Though uniqueness of the steady solution is not proven rigorously, all steady solutions found by numerical iteration yielded the same result, independent of the initial geometry.

Using similar arguments, we show the non-existence of a solution (minimizer) in the free-margin case. This non-existence result follows from the same elevation/accumulation feedback 'instability' identified in earlier literature (Bodvarsson, 1955; Weertman, 1961), but unlike those sources we show non-existence in a model which includes ice deformation and which uses a realistic mass balance (linearly dependent on altitude and not piecewise-constant).

Another feature observed in the shallow-ice-sheet solutions is unbounded surface inclination at the margins. This alone may not be unrealistic for advancing ice tongues or even steady termini. However, the basal shear stress in the SIA is unbounded at the margins for some solutions (e.g. the Vialov profile; Vialov, 1958), or it takes a nonzero value in some other solutions (e.g. Bueler, 2003). Without longitudinal coupling, the basal value of the driving shear stress, which is proportional to the product of the local surface inclination and ice thickness, should vanish. Otherwise, 


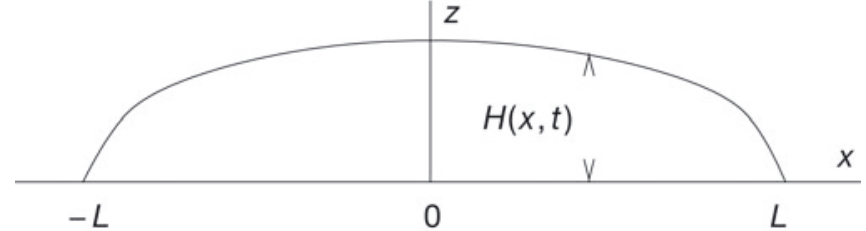

Fig. 1. Idealized two-dimensional ice sheet.

a non-sliding terminus with vertical end point should be shearing and eventually become overhanging, which is not consistent with steady state.

In this paper we solve the shallow-ice-sheet equation with power-law-type sliding parameterization independently using two different numerical methods: (1) a finite-difference scheme to solve the time-dependent partial differential equation and (2) a numerical quadrature to solve the ODE for the steady state. The transient solution evolved into a steady state where the basal shear stress vanishes at an apparently vertical terminus.

We present the model in the next section and give the existence theorem in the 'Mathematical analysis' section. We then present the numerical methods and the results of numerical simulations. Details of the mathematical proofs and solutions are given in the appendices.

\section{GOVERNING EQUATIONS}

We consider the evolution of an idealized two-dimensional ice sheet on a flat base, centred at the origin (Fig. 1). The ice domain is described by the ice-thickness function, $H(x, t)$, at point $x$ and time $t$ :

$$
H:[-L, L] \times[0, T] \longrightarrow \mathbb{R}_{+},
$$

where $L>0$ limits the horizontal and $T>0$ the vertical extent of the domain and $\mathbb{R}_{+}$denotes the set of positive real numbers. Obviously, the ice thickness, $H$, must be positive.

Ice is considered to be a non-Newtonian fluid governed by Glen's flow law (Glen, 1958). In the SIA, the horizontal component of the ice velocity is given by

$$
u=-2 A(\rho|\mathbf{g}|)^{n} \frac{\partial H}{\partial x}\left|\frac{\partial H}{\partial x}\right|^{n-1} \int_{0}^{z}(H-\xi)^{n} \mathrm{~d} \xi+u_{\mathrm{b}}
$$

where $\mathbf{g}$ is gravity, $\rho$ is the density, $\xi$ is a variable of integration and $u_{\mathrm{b}}$ is the basal velocity. The rate factor, $A$, and the flowlaw exponent, $n>1$, represent the flow properties of ice and are assumed to be constant.

Sliding over a hard rough bed is mostly determined by the deformation of the ice across the roughness elements. Dimensional analysis and model calculations suggest that the mean basal velocity is then a power function of the basal shear stress with the same exponent, $n$, as in the flow law for ice (Weertman, 1957, 1964; Gudmundsson, 1997; Greve and Blatter, 2009),

$$
u_{\mathrm{b}}=C\left|\tau_{\mathrm{b}}\right|^{n-1} \tau_{\mathrm{b}}
$$

where $\tau_{\mathrm{b}}$ is the basal shear stress in the SIA,

$$
\tau_{\mathrm{b}}=-\rho|\mathbf{g}| H \frac{\partial H}{\partial x}
$$

and $C \geq 0$ is here assumed to be constant. Combining Equations (2) and (3) yields

$$
u_{\mathrm{b}}=-C(\rho|\mathbf{g}|)^{n} H^{n} \frac{\partial H}{\partial x}\left|\frac{\partial H}{\partial x}\right|^{n-1} .
$$

Consideration of mass continuity for a vertical column (Paterson, 1994; Picasso and others, 2004) yields the SaintVenant equation:

$$
\frac{\partial H}{\partial t}+\frac{\partial}{\partial x}\left(\int_{0}^{H(x, t)} u \mathrm{~d} z\right)=b(x, H(x, t), t),
$$

where $b=b(x, z, t)$ is the accumulation (mass-balance) function. Combining Equations (1), (4) and (5) yields

$\frac{\partial H}{\partial t}-\Gamma \frac{\partial}{\partial x}\left[(H+\bar{C}) H^{n+1}\left|\frac{\partial H}{\partial x}\right|^{n-1} \frac{\partial H}{\partial x}\right]=b(x, H(x, t), t)$,

with the two positive constants

$$
\Gamma=\frac{2(\rho|\mathbf{g}|)^{n} A}{n+2}, \quad \bar{C}=\frac{C(n+2)}{2 A},
$$

where $\Gamma$ is related to ice viscosity and $\bar{C}$ to basal sliding.

Given the initial ice thickness function, $H_{0}$, the timedependent problem consists of finding $H$ such that:

$$
\begin{aligned}
H \text { satisfies Equation (6) } & \text { if } H>0, \\
H=0 & \text { otherwise, } \\
H(t,-L)=H(t, L)=0, & t \in(0, T), \\
H(0, x)=H_{0}(x), & x \in(-L, L) .
\end{aligned}
$$

The existence of and the uniqueness of the solution for the system of Equations (8-11) has been investigated and proved by Calvo and others (2002) for a mass balance which is independent of the altitude.

\section{MATHEMATICAL ANALYSIS}

In this section we investigate the existence of stationary solutions if the mass balance, $b$, increases linearly with the altitude,

$$
b(x, z, t)=a_{\mathrm{m}}\left(z-z_{\mathrm{ELA}}\right),
$$

where $a_{\mathrm{m}}>0$ is the vertical melt gradient and $z_{\mathrm{ELA}}>0$ is the equilibrium-line altitude. The basal sliding is neglected, for now, by assuming $C=0$. The stationary problem then consists of finding $H:[-L, L] \mapsto \mathbb{R}$ such that:

$$
\begin{array}{r}
-\Gamma\left(H^{n+2}\left|H^{\prime}\right|^{n-1} H^{\prime}\right)^{\prime}=a_{\mathrm{m}}\left(H-z_{\mathrm{ELA}}\right) \quad \text { if } H>0, \\
H=0 \quad \text { otherwise, } \\
H(-L)=H(L)=0 .
\end{array}
$$

Here $^{\prime}$ denotes the spatial derivative with respect to $x$.

\section{Scaling}

The previous problem is rewritten for the horizontal interval $[-1,1]$ with a scaling of $H$ and $x$,

$$
\widetilde{H}(\widetilde{x})=\frac{H(x)}{Z_{\mathrm{ELA}}} \quad \text { with } \quad \widetilde{x}=\frac{x}{L} .
$$

Equation (13) is reformulated in terms of $\widetilde{H}$ and $\widetilde{x}$ :

$$
-\Gamma \frac{Z_{\mathrm{ELA}}^{2 n+1}}{L^{n+1}}\left(\widetilde{H}^{n+2}\left|\widetilde{H}^{\prime}\right|^{n-1} \widetilde{H}^{\prime}\right)^{\prime}=a_{\mathrm{m}}(\widetilde{H}-1),
$$


where' now denotes the derivative with respect to $\widetilde{x}$. We transform Equation (16) into a $p$-Laplacian equation by applying a power transformation (Raviart, 1970; Calvo and others, 2002; Antontsev and de Oliveira, 2008),

$$
\eta=\widetilde{H}^{\frac{2(n+1)}{n}},
$$

which yields:

$$
-\left(\left|\eta^{\prime}\right|^{n-1} \eta^{\prime}\right)^{\prime}=\alpha\left[\eta^{\frac{n}{2(n+1)}}-1\right],
$$

where

$$
\alpha=\frac{1}{\Gamma} \frac{L^{n+1}}{z_{\mathrm{ELA}}^{2 n+1}}\left[\frac{2(n+1)}{n}\right]^{n} a_{\mathrm{m}}
$$

is a positive dimensionless coefficient. Finally, the problem of Equations (13-15), reformulated in terms of $\eta$, consists of finding a function $\eta:[-1,1] \mapsto \mathbb{R}$ such that

$$
\begin{aligned}
\eta \text { satisfies Equation (18) } & \text { if } \eta>0, \\
\eta=0 & \text { otherwise, } \\
\eta(-1)=\eta(1)=0 . &
\end{aligned}
$$

Equations (20-22) form an obstacle problem which allows the solution to be identically zero on a subset of $(-1,1)$. In particular, the function vanishing on $(-1,1)$ is a trivial solution.

The goal of the next subsection is to prove the existence of a smooth function, $\eta>0$, on $(-1,1)$ satisfying Equations (20-22). The existence of a strictly positive solution, $H$, of the original obstacle problem, Equations (13-15), follows immediately by undoing the power transformation.

\section{Existence of solutions with fixed margins}

The existence of positive solutions to problems of the form

$$
-\left(\left|\eta^{\prime}\right|^{p-2} \eta^{\prime}\right)^{\prime}=g(\eta)
$$

in $(-1,1)$, with boundary conditions $\eta(-1)=\eta(1)=0$, where $g$ is a sign-changing nonlinear term, has been the topic of recent publications, including Lü and others (2005). In our case $p=n+1$. The function $g: \mathbb{R}_{+} \longrightarrow \mathbb{R}$ defined as

$$
g(\eta)=\alpha\left[\eta^{\frac{n}{2(n+1)}}-1\right]
$$

and appearing in Equation (18), does not, however, satisfy the technical assumptions required in theorem 3.1 of Lü and others (2005).

In what follows, we overcome this difficulty and prove the existence of strictly positive, even and radially decreasing solutions to the problem of Equations (20-22) using the calculus of variations in theorem 2 (detailed below). Several steps are needed to reach this objective. In step 1, we consider an associated minimization problem and we prove the existence of a minimizer. In step 2, we show that the minimizer can be chosen even, radially decreasing and strictly positive on $(-1,1)$, as long as $\alpha$ is large enough. In step 3, we show that this minimizer solves the problem (Equations (20-22)).

Throughout this subsection, we use $L^{p}(-1,1)$ to denote the space of all $p$ th power Lebesgue-integrable functions on $(-1,1)$, and we denote by $W^{1, p}(-1,1)$ the space of all functions $\eta \in L^{P}(-1,1)$ admitting a weak derivative $\eta^{\prime}$ in $L^{P}(-1,1)$ (Lieb and Loss, 1997).

\section{Step 1: minimization problem}

From the point of view of the calculus of variations, any solution of Equation (18) is a stationary point (i.e. a point that makes the derivative zero) of a carefully chosen functional (Evans, 1999; Dacorogna, 2008). The problem of Equations (20-22) is then re-interpreted as a minimization problem, since any minimum is a stationary point. We will show this minimization problem has at least one solution, a global minimum. We cannot exclude that in some cases there are additional local minima, and possibly additional stationary points which are not local minima. The arbitrary choice of a minimum, versus a maximum, is guided by mathematical usage, since many physical problems can be rewritten in the form of an energy minimization.

In order to analyse the problem (Equations (20-22)), we introduce the following functional space for our fixed-margin problem:

$$
W_{0}^{1, p}(-1,1)=\left\{\eta \in W^{1, p}(-1,1) ; \quad \eta(-1)=\eta(1)=0\right\},
$$

where $p=n+1$. Note that since $p>2(n>1)$, all functions of $W_{0}^{1, p}(-1,1)$ are continuous on $[-1,1]$ (e.g. Brezis, 1999).

Since Equation (18) makes sense only where $\eta$ is positive, we consider a convex subset, $K$, of $W_{0}^{1, p}(-1,1)$, defined by

$$
K=\left\{\eta \in W_{0}^{1, p}(-1,1): \quad \eta \geq 0\right\} .
$$

We introduce the functional $J$, defined on $K$,

$$
J(\eta)=\int_{-1}^{+1}\left(\frac{1}{p}\left|\eta^{\prime}\right|^{p}-\frac{\alpha}{q} \eta^{q}+\alpha \eta\right) \mathrm{d} x,
$$

where $q=\frac{n}{2(n+1)}+1 \in\left(\frac{5}{4}, \frac{3}{2}\right)$. The functional, $J$, is differentiable at any input, $\eta$, such that $\eta>0$ on $(-1,1)$. Its derivative, $\left\langle J^{\prime}(\eta), \varphi\right\rangle$, defined by

$$
\left\langle J^{\prime}(\eta), \varphi\right\rangle=\lim _{\varepsilon \rightarrow 0} \frac{J(\eta+\varepsilon \varphi)-J(\eta)}{\varepsilon},
$$

measures the infinitesimal variation of $J$ at point $\eta$ following the direction $\varphi$. A formal derivation of Equation (23) leads to

$$
\left\langle J^{\prime}(\eta), \varphi\right\rangle=\int_{-1}^{+1}\left(\left|\eta^{\prime}\right|^{p-2} \eta^{\prime} \varphi^{\prime}-\alpha \eta^{q-1} \varphi+\alpha \varphi\right) \mathrm{d} x
$$

Note that

$$
\left\langle J^{\prime}(\eta), \varphi\right\rangle=0 \quad \text { for all functions } \varphi
$$

corresponds exactly to the weak formulation of Equation (18), with boundary conditions given by Equation (22), that is obtained by multiplying Equation (18) by an arbitrary test function, $\varphi$, and integrating by parts.

To prove the existence of a minimizer, the functional, $J$, needs to satisfy several mathematical properties, mainly coerciveness and convexity in the variable $\eta^{\prime}$. Using standard arguments of convex analysis, using the convexity of the function $\xi \mapsto \frac{1}{p}|\xi|^{p}$, we are able to state the existence of a minimizer in the next theorem. The complete proof is given in Appendix A.

Theorem 1 There exists at least one $\bar{\eta} \in K$ such that $J(\bar{\eta}) \leq$ $J(\eta)$ for all $\eta \in K$.

Nevertheless, our understanding of the calculus of variations, including consideration of the arguments of Dacorogna (2008), fails to resolve the uniqueness of $\bar{\eta}$ because the function $\eta \mapsto-\frac{\alpha}{q} \eta^{q}+\alpha \eta$ is not convex. 


\section{Step 2: properties of the minimizer}

We formulate three lemmas stating the properties of the minimizer of theorem 1. They are proven in Appendix A. The first lemma shows that any minimizer of $J$ in $K$ may be chosen even and radially decreasing. More precisely, if $\bar{\eta}$ is a minimizer of $J$ in $K$, then we consider the even decreasing rearrangement (also called Schwarz symmetrization) $\eta^{*}$ of $\bar{\eta}$ (Lieb and Loss, 1997). The function $\eta^{*}$ remains in $K$ and satisfies $J\left(\eta^{*}\right) \leq J(\bar{\eta})$. We deduce the following lemma.

Lemma 1 There exists an even and radially decreasing function on $(0,1)$ in the set $K$ called $\eta^{*}$ such that $J\left(\eta^{*}\right) \leq J(\eta)$ for all $\eta \in K$.

The next lemma states that there exists $\eta \in K$ such that $J(\eta)<0$, as long as $\alpha$ is large enough. As a consequence, $J\left(\eta^{*}\right)<0$ and $\eta^{*}$ is not the zero function.

Lemma 2 There exists $\alpha_{0} \geq 0$ such that if $\alpha>\alpha_{0}$, then $J\left(\eta^{*}\right)<0$, where $\eta^{*}$ is obtained in lemma 1.

The threshold, $\alpha_{0}$, given in lemma 2, has the following physical meaning: the coefficient $\alpha$, defined by Equation (19), needs to be sufficiently big (equivalently, $L$ needs to be big enough, or $Z_{\mathrm{ELA}}$ small enough, or $a_{\mathrm{m}}$ big enough) to guarantee the existence of a nonzero function, $\eta^{*}$, and then the existence of a steady ice sheet. It should be stressed that $\alpha_{0}$ depends on $n$ but does not depend on $L, z_{\mathrm{ELA}}, a_{\mathrm{m}}$ or $\Gamma$.

From these two lemmas, the function, $\eta^{*}$ is even, nonzero and decreasing on $(0,1)$. Let $\gamma \in[0,1]$ be the threshold, such that $\eta^{*}(x)>0$ for all $x \in[0, \gamma)$ and $\eta^{*}(x)=0$ for all $x \in[\gamma, 1]$. The third lemma states that the scaled function $\zeta(x)=\gamma^{\frac{1-p}{p}} \eta^{*}(\gamma x)$ of $\eta^{*}$ that belongs to $K$ satisfies $J(\zeta)<J\left(\eta^{*}\right)$ if $\gamma<1$. As a consequence, $\gamma$ is necessarily equal to 1 and $\eta^{*}>0$ on $(-1,1)$.

Lemma 3 Let $\eta^{*} \in K$ be the even and radially decreasing function on $(0,1)$ obtained in lemma 1. Suppose that $\alpha>\alpha_{0}$, where $\alpha_{0}$ is given in lemma 2. Then $\eta^{*}>0$ on $(-1,1)$.

The first variation (Equation (24)) of $J$ at point $\eta^{*}$ is now well defined, since $\eta^{*}>0$ on $(-1,1)$.

\section{Step 3: back to problem, Equations (20-22)}

By using a first-variation argument, we are able to show that any minimizer of $J$ which is strictly positive on $(-1,1)$ is a solution of Equations (20-22) as long as $\alpha$ is large enough (see the proof in Appendix A). So we obtain the following result.

Theorem 2 There exists $\alpha_{0}>0$ and for $\alpha>\alpha_{0}$ there exists a function $\eta^{*} \in K$ which is even, radially decreasing and strictly positive on $(0,1)$ satisfying Equations (20-22). Moreover $\eta^{*}$ is continuously differentiable on $[-1,1]$.

By theorem 2, there exists a function

$$
H(x)=Z_{\mathrm{ELA}}\left[\eta^{*}(x / L)\right]^{\frac{n}{2(n+1)}}
$$

even, radially decreasing, strictly positive on $(0,1)$ and continuously differentiable on $(-1,1)$, that satisfies the original problem, Equations (13-15).

\section{Non-existence with free margins}

The space $W_{0}^{1, p}(-1,1)$ used in the existence results above includes fixed-margin boundary conditions. Lemma 3 shows that if $\eta(x)$ has its margin at $\gamma<1$ then 'advancing' the margin to $\gamma=1$ lowers the value of the functional, $J$, so the steady solutions found above are positive in $(-1,1)$ and also $\eta( \pm 1)=0$. These fixed-margin solutions correspond to ice sheets with nonzero flux at $x= \pm L$, however.

Removing the fixed-margin boundary condition, $\eta( \pm 1)=$ 0 , we consider $\eta$ with arbitrary support in $W^{1, p}(-\infty, \infty)$. Let $\widehat{K}=\left\{\eta \in W^{1, p}(-\infty, \infty): \eta \geq 0\right\}$. Also let

$$
\widehat{\jmath}(\eta)=\int_{-\infty}^{\infty}\left(\frac{1}{p}\left|\eta^{\prime}\right|^{p}-\frac{\alpha}{q} \eta^{q}+\alpha \eta\right) \mathrm{d} x,
$$

which is the same functional extended to the whole real line. Then $\widehat{\jmath}$ is unbounded below, by the following result (proved in Appendix A).

Lemma 4 There exists a sequence of even, radially decreasing functions, $\widehat{\eta}_{k} \in \widehat{K}$, each supported in a finite interval, so that $\lim _{k \rightarrow \infty} \widehat{\jmath}\left(\widehat{\eta}_{k}\right)=-\infty$.

Because the functional values for these profiles, $\widehat{\jmath}\left(\widehat{\eta}_{k}\right)$, are exactly computable, and because these values are unboundedly negative, we know that there is no steady solution which could minimize $\hat{\jmath}$.

The ice sheets corresponding to $\widehat{\eta}_{k}$ can be compared to the states of a time-dependent solution undergoing runaway growth, in both horizontal and vertical extent. Such a timedependent solution could grow by adding accumulation with increasing surface elevation, but at the same time flow would be insufficient to eliminate mass in the ablation area near the margin (cf. Weertman, 1961). By contrast, in the freemargin case with mass balance, $b(x)$, depending only on horizontal position and with sufficient ablation outside a finite support accumulation interval so that $\int_{-\infty}^{\infty} b(x) \mathrm{d} x<0$, the functional $\widehat{\jmath}$ would be bounded below.

\section{NUMERICAL METHOD}

A simple way to obtain a numerical solution of the problem given by Equations (13-15) is to implement that given by Equations (8-11) and seek transient solutions converging into steady states. In this scheme, a time-step is one step in a fixed-point iteration scheme. Rewriting Equation (6) in the form

$$
\frac{\partial H}{\partial t}-\Gamma \frac{\partial}{\partial x}\left(D \frac{\partial H}{\partial x}\right)=b
$$

where

$$
D=(H+\bar{C}) H^{n+1}\left|\frac{\partial H}{\partial x}\right|^{n-1},
$$

Equation (28) can be classified as a 'nonlinear diffusion equation', where $D$ is the diffusivity. The nature of Equation (28) and the simplicity of the geometry suggests a finite-difference scheme on a uniform mesh be used in its solution. This problem has been widely investigated and several schemes have been proposed (Huybrechts and others, 1996; Van der Veen, 1999; Bueler and others, 2005; Van den Berg and others, 2006). The existing schemes can be characterized by two features. The first concerns the way to approximate the diffusivity, D, (Huybrechts and others, 1996; Van der Veen, 1999) while the second concerns the level of implicitness of the numerical scheme. We opt for an approximation of $D$ at the midpoint of the discretization grid, based on centred mean ice thickness, $H$, and local surface derivative, $\partial H / \partial x$. This choice corresponds to 'type I' described by Huybrechts and others (1996). We use a semi-implicit time discretization 
of the diffusive part of Equation (28): $\partial H / \partial x$ is approximated at the current time while $D$ is approximated at the previous time. In the case of an elevation-dependent mass balance (e.g. Equation (12)) $b$ is updated at each time-step using the elevation at the previous time (Van den Berg and others, 2006). Under appropriate stability conditions, this finitedifference scheme is locally order one accurate in time and order two accurate in space.

\section{NUMERICAL RESULTS}

In this section, we present two stationary solutions of the shallow-ice-sheet equation: case 1 for a mass balance linearly increasing with elevation and case 2 for an ice sheet with Weertman-type sliding.

\section{Case 1: altitude-dependent mass balance}

In case 1 we aim to find a numerical stationary strictly positive solution to the problem, Equations (13-15), the existence of which has been proved in theorem 2. In all cases, the physical ice flow parameters are chosen as $n=3$ and $A=3.17 \times 10^{-24} \mathrm{~Pa}^{-3} \mathrm{~s}^{-1}$.

We use the mass-balance parameterization of Equation (12) with $a_{\mathrm{m}}=3.0 \times 10^{-4} \mathrm{a}^{-1}$ and $z_{\mathrm{ELA}}=1000 \mathrm{~m}$. Numerical tests are performed with three initial conditions:

(1) $H_{0}(x)=2 z_{\mathrm{ELA}} \cos \left(\frac{\pi}{2 L} x\right)$,

(2) $H_{0}(x)=6 z_{\mathrm{ELA}} \cos \left(\frac{\pi}{2 L} x\right)$,

(3) $H_{0}(x)=z_{\mathrm{ELA}} \cos \left(\frac{\pi}{2 L} x\right)+z_{\mathrm{ELA}}\left[\cos \left(\frac{6 \pi}{L} x\right)\right]^{2}$,

where $L=1000 \mathrm{~km}$. Ice is assumed to be fixed at the base, i.e. $C=0$. The time-step is 1 year and the interval $(-L, L)$ is discretized by 50 points.

By initializing the simulation with conditions 1 and 2, we check the uniqueness of the numerical solution (Fig. 2). Note that the shape stabilizes faster with initial condition 2 than with initial condition 1. Indeed, initial condition 1 is far from the steady solution, and the accumulation/ablation process needs $\sim 8000$ years to reach a comparable volume to the steady shape. Initial condition 2 is closer to the solution in terms of volume, and the diffusion (which acts faster than the accumulation/ablation step) allows the stationary solution to be reached quickly. With the fluctuating initial condition 3, the diffusion becomes smooth quickly and passes through transient shapes, comparable to condition 1, to the same steady state. Several non-symmetric shapes were initialized (not shown); all resulted in the same symmetric steady-state shape.

Note that the stationary solution necessarily satisfies the boundary conditions implicit in lemma 3 . Indeed the $z$ dependent mass balance induces a positive feedback of the solution in the source term of Equation (6). Runaway growth of the ice sheet occurs as $L \rightarrow \infty$, i.e. with unlimited free margins.

The regularity of $H$ is an important feature of the solution given by theorem 2. Figure 3 displays the numerical spatial derivative of the solution obtained with two different levels of resolution (100 and 400 points of discretization). For increasing resolution of the grid, the derivative converges to a continuous function, however, with a vertical asymptote at zero.

The time evolution of the functional, J, applied to timedependent inputs $\eta(t, \cdot)=\left(H(t, \cdot) / z_{\mathrm{ELA}}\right)^{\frac{2(n+1)}{n}}$, is shown in
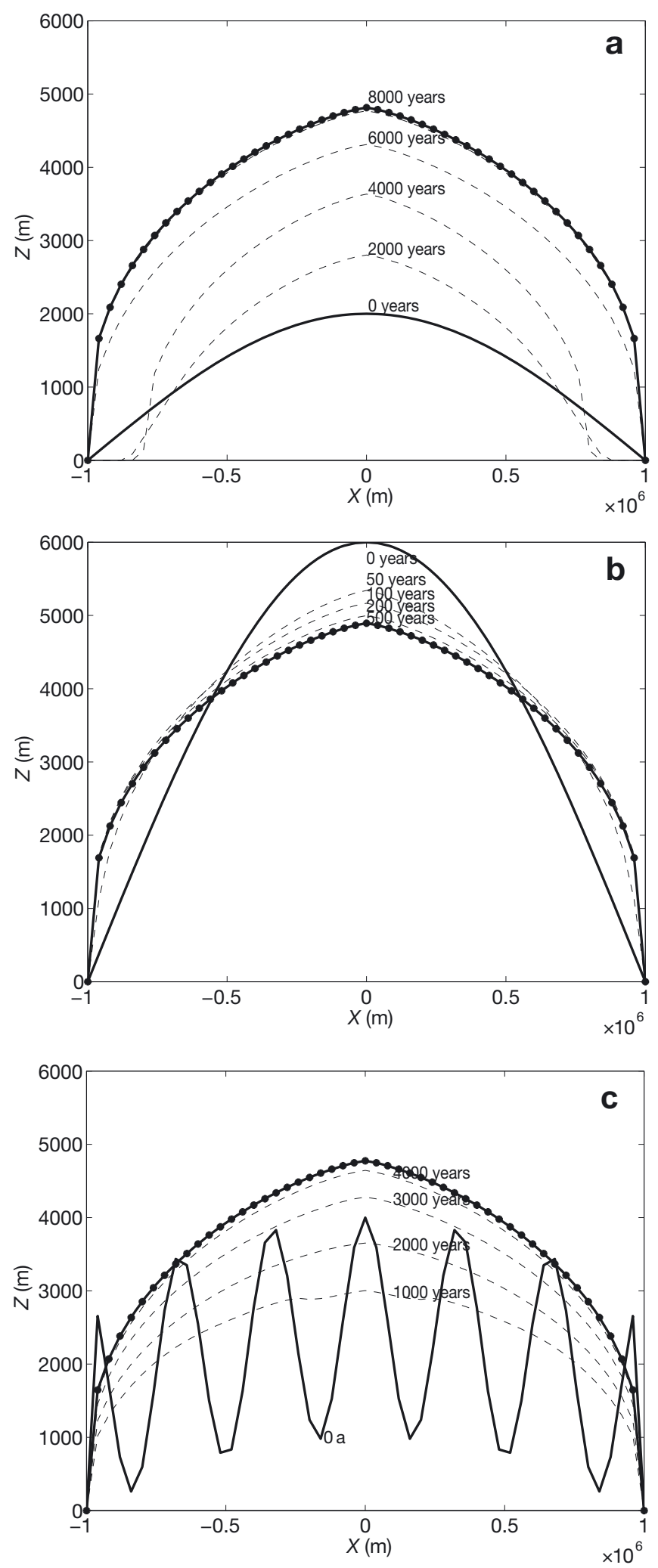

Fig. 2. Experiments with different initial shapes: (a) initialization 1, (b) initialization 2 and (c) initialization 3. The time evolution of the ice thickness function is shown, with the initial shape as a continuous curve, the transient states as dotted curves and the steady state as a continuous curve with black dots.

Figure 4. As expected, the functional, J, decreases and reaches a negative value at the limit, in accordance with lemma 2. Each initial function, $H_{0}$, must be chosen thick 


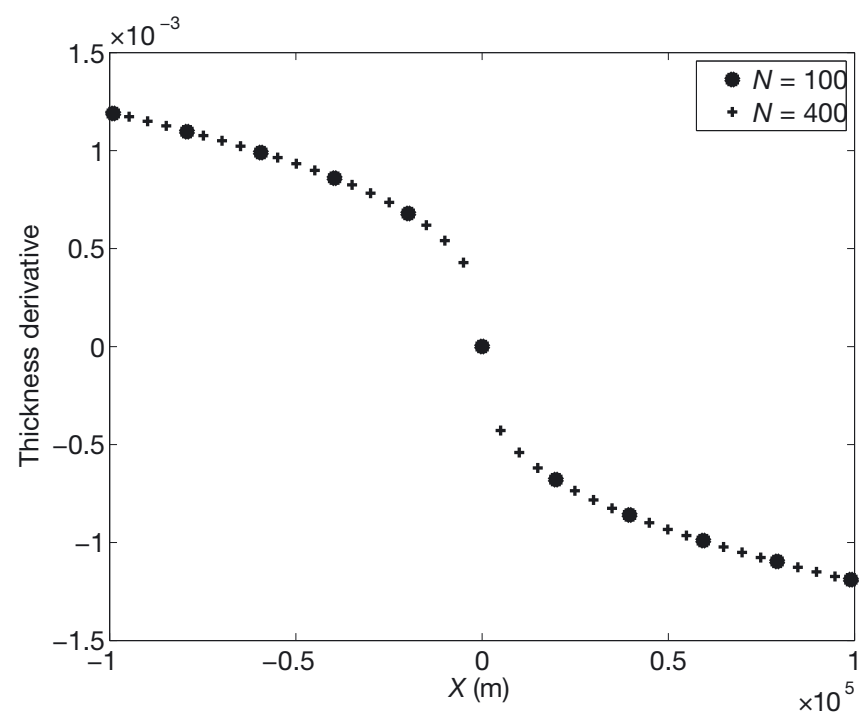

Fig. 3. Stationary thickness derivative function for two mesh resolutions, $N=100$ and $N=400$, where $N$ is the number of points of discretization.

enough, otherwise the diffusion step could drive transient solutions below $z_{\text {ELA }}$ that would imply the inescapable collapse of the solution. The choice of a safe initial function has an interpretation in terms of the functional, J. Note that the zero function locally minimizes J. If the initial function is too close to zero, then the transient numerical solution, which minimizes J over time, converges to this local minimum and then fails to catch the global minimum.

The particular choices of $L, Z_{\mathrm{ELA}}, a_{\mathrm{m}}, n$ and $A$ fix the value of $\alpha$ (see Equation (19)). Since the numerical steady shape is positive, then $\alpha>\alpha_{0}$, where $\alpha$ is given in lemma 2. We are able to check the existence of this threshold. Indeed, by reducing $L$ from 1000 to $100 \mathrm{~km}$, we also reduce $\alpha$. In this configuration, the stationary numerical solution vanishes everywhere on $(-L, L)$ and the zero function is the minimum of $J$, which is consistent with lemma 2 . An empirical value of $\alpha_{0}$ can be estimated by repeating the calculation using various values of $\alpha$. When $n=3$, we find $\alpha_{0} \sim 147$. The

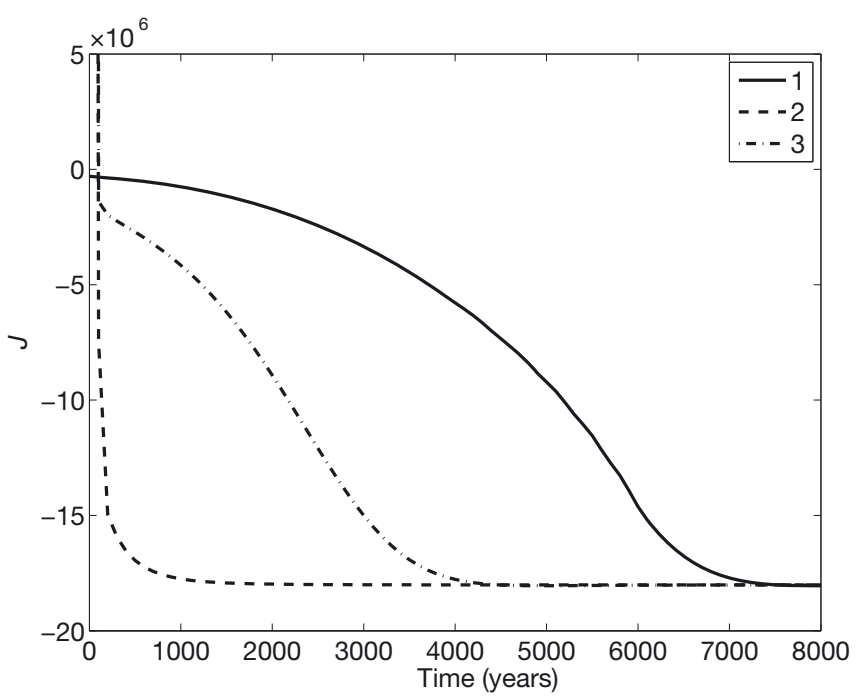

Fig. 4. Time evolution of the functional, $J$, for each experiment corresponding to the initial shapes: 1, 2 and 3 .

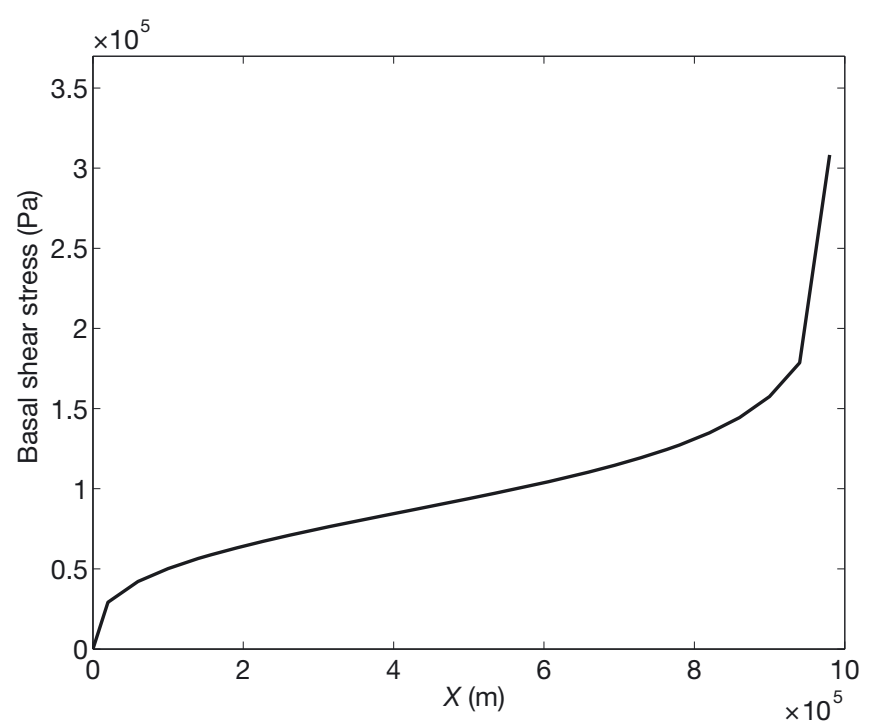

Fig. 5. Basal shear stress, $\tau_{\mathrm{b}}$, corresponding to the steady-state shape in case 1.

physical parameters $L, Z_{\mathrm{ELA}}, a_{\mathrm{m}}$ and $A$ need to satisfy the inequality $\alpha>\alpha_{0}$ to ensure the existence of a nonzero steady shape.

This numerical evidence suggests the existence of the solution proved in theorem 2 and the uniqueness of this even, positive and radially decreasing steady shape. Note that the basal shear stress, $\tau_{\mathrm{b}}$, of such a steady-state shape is not bounded and goes to infinity when $x$ goes to the margins (Fig. 5).

\section{Case 2: altitude-independent mass balance and sliding}

In case 2, a mass-balance function is chosen that does not depend on the unknown elevation of the ice sheet. This allows us to compute the location of the steady-state margin independently of the numerical solution of the ice-sheet shape. We choose a mass-balance function with constant accumulation in the inner part and constant ablation in the marginal part (Paterson, 1994). The mass balance is defined by:

$$
b(x)= \begin{cases}a_{1}, & \text { if } x \in[-\bar{L}, \bar{L}], \\ a_{2}, & \text { else, }\end{cases}
$$

with $a_{1}=5 \mathrm{ma}^{-1}, a_{2}=-10 \mathrm{~m} \mathrm{a}^{-1}$ and $\bar{L}=500 \mathrm{~km}$. The model domain extends to $L=1000 \mathrm{~km}$. The steady-state margin is at $x=750 \mathrm{~km}$, well inside the model domain.

The stationary equation corresponding to Equation (6) can be integrated by numerical quadrature, up to a change of variable, which is a more accurate numerical scheme than the finite-difference ODE approach. Details, including a new power transformation to make a well-behaved ODE in the sliding case, are given in Appendix B.

In seeking to avoid such behaviour, basal sliding is introduced. With no sliding, the transient solutions converge to the steady-state solution of the stationary equation. They have non-vanishing basal shear stress at the margin (Greve and Blatter, 2009).

The steady shapes are shown in Figure 6, both for vanishing sliding and for sliding with a coefficient $\bar{C}=50000$. In contrast to case 1 , the extent of the resulting steady-state ice 


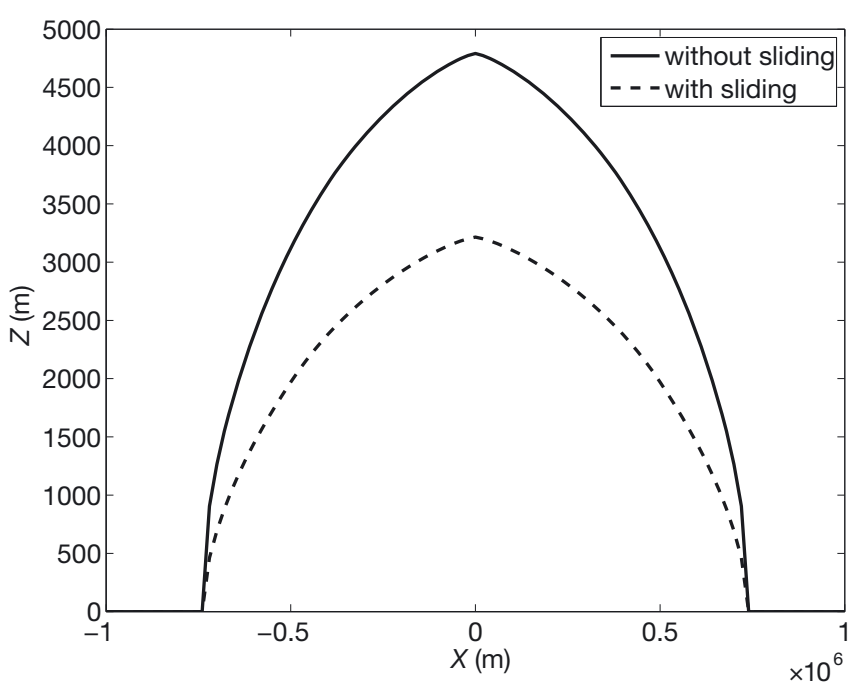

Fig. 6. Steady-state shape in case 2, without and with sliding.

sheet does not fill the given domain $[-L, L]$ and the margin position matches the theoretical position accurately. Figure 7 shows the basal shear stress of the steady-state shape for both non-sliding and sliding ice sheets. For any sliding coefficient, $\bar{C}>0$, the shear stress drops to zero at the margin.

\section{CONCLUSIONS AND DISCUSSION}

We have proven the existence of solutions of the stationary shallow-ice-sheet equation for a flat bed, vanishing sliding and fixed-margin position, in the case where the mass balance is a linear function of the unknown elevation. A change of variables allows us to rewrite the equation in the form of a $p$-Laplacian. Any minimizer of a corresponding functional, $J$, solves this equation. Using a symmetrization technique, the existence of an even, radially decreasing and strictly positive minimizer (solution) has been shown. Due to a lack of convexity, uniqueness of the solution needs to be investigated by a different approach. Essentially the same tools allow us to show the non-existence of solutions with free margin position. The interpretation of $J$ as a system 'energy' is an aspect still to be investigated.

Removing the flat-bed or the non-sliding assumption changes the mathematical problem in a fundamental way. The power transformation, $\eta=\widetilde{H}^{2(n+1) / n}$, used to transform the non-sliding flat-bed case of the SIA into a $p$-Laplacian problem (Raviart, 1970; Calvo and others, 2002) does not remove the double nonlinearity if the bed is not flat or if sliding is included. Further research is needed to resolve basic existence questions with sliding and/or non-flat bed, even in simpler cases where mass balance is independent of surface elevation.

A finite-difference scheme for time-evolving solutions was used to find steady-state solutions numerically. The numerical experiments confirmed the theoretical results and suggested the existence of only one positive stable solution with fixed margins.

Similarly to the Vialov solution (Greve and Blatter, 2009), the net mass balance is nonzero for fixed-margin solutions found here, and correspondingly the mass flux does not drop to zero at the margin. Thus the fixed-margin solution is not a steady-state solution for free-margin ice sheets. Runaway growth of the ice sheet occurs with free margins for large

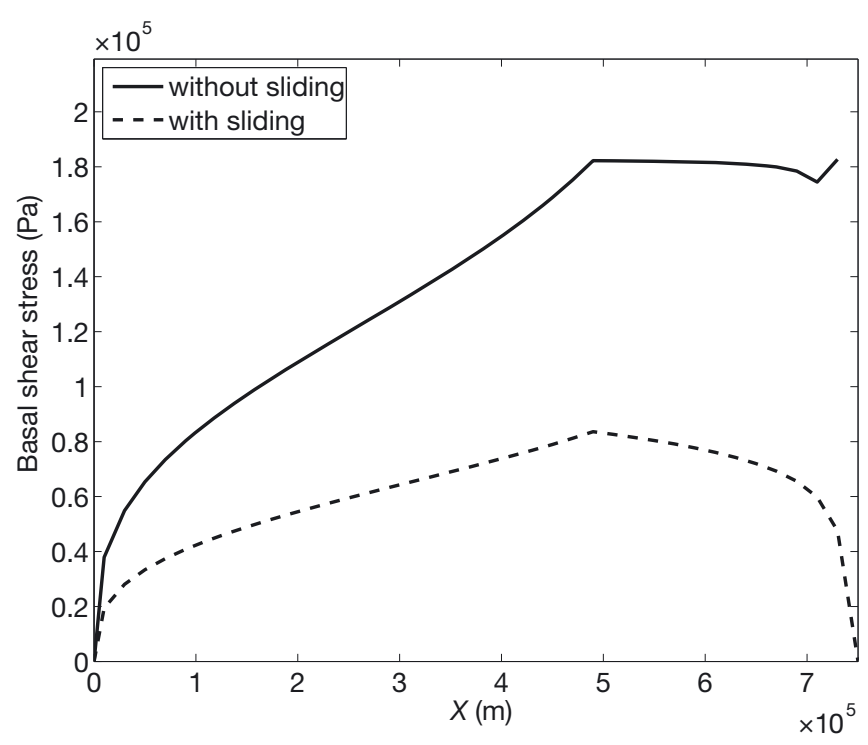

Fig. 7. Basal shear stress, $\tau_{b}$, corresponding to the steady-state shape in case 2 , without and with sliding.

enough initial geometries. If the initial shape leads to a shrinking ice volume, no steady-state shape exists other than the zero solution. This shows the existence of an unstable equilibrium between runaway growth and runaway shrinkage.

The solution of the shallow-ice-sheet equation exhibits realistic physical conditions at the margin when Weertman type of sliding is included, in a case with accumulation only depending on the horizontal coordinate. Although the marginal surface still seems to have unbounded slope, the basal shear stress, and consequently the sliding velocity, vanish at the margin, giving conditions consistent with a steady-state geometry.

\section{ACKNOWLEDGEMENTS}

This work was funded by the Swiss National Science Foundation, project No. 200021-119721. E.B. received support from NASA grant NNX09AJ38G. We appreciate the comments of editor R. Greve, an anonymous reviewer and J. Bassis, all of which improved the paper and helped us place it properly in context.

\section{REFERENCES}

Antontsev, S.N. and H.B. de Oliveira. 2008. Qualitative properties of the ice-thickness in a 3D model. WSEAS Trans. Math., 7(3), $78-86$.

Bodvarsson, G. 1955. On the flow of ice-sheets and glaciers. Jökull, $5,1-8$.

Brezis, H. 1999. Analyse fonctionnelle: théorie et applications. Paris, Éditions Dunod.

Brock, F. 2000. Continuous rearrangement and symmetry of solutions of elliptical problems. Proc. Indian Acad. Sci. (Math. Sci.), 110(2), 157-204.

Bueler, E. 2003. Construction of steady state solutions for isothermal shallow ice sheets. Fairbanks, AK, University of Alaska Fairbanks. Department of Mathematics and Statistics. (UAF DMS Tech. Rep. 03-02.)

Bueler, E., C.S. Lingle, J.A. Kallen-Brown, D.N. Covey and L.N. Bowman. 2005. Exact solutions and verification of numerical models for isothermal ice sheets. J. Glaciol., 51(173), 291-306. 
Bueler, E., J. Brown and C. Lingle. 2007. Exact solutions to the thermomechanically coupled shallow-ice approximation: effective tools for verification. J. Glaciol., 53(182), 499-516.

Calvo, N., J.I. Díaz, J. Durany, E. Schiavi and C. Vázquez. 2002. On a doubly nonlinear parabolic obstacle problem modelling ice sheet dynamics. SIAM J. Appl. Math., 63(2), 683-707.

Dacorogna, B. 2008. Direct methods in the calculus of variations. Second edition. New York, Springer.

Evans, L.C. 1999. Partial differential equations. Providence, RI, American Mathematical Society.

Glen, J.W. 1958. The flow law of ice: a discussion of the assumptions made in glacier theory, their experimental foundation and consequences. IASH Publ. 47 (Symposium at Chamonix 1958 - Physics of the Movement of the Ice), 171-183.

Greve, R. and H. Blatter. 2009. Dynamics of ice sheets and glaciers. Dordrecht, etc., Springer.

Gudmundsson, G.H. 1997. Basal-flow characteristics of a nonlinear flow sliding frictionless over strongly undulating bedrock. J. Glaciol., 43(143), 80-89.

Halfar, P. 1981. On the dynamics of the ice sheets. J. Geophys. Res., 86(C11), 11,065-11,072.

Halfar, P. 1983. On the dynamics of the ice sheets 2. J. Geophys. Res., 88(C10), 6043-6051.

Huybrechts, P., T. Payne and the EISMINT Intercomparison Group. 1996. The EISMINT benchmarks for testing ice-sheet models. Ann. Glaciol., 23, 1-12.

Lieb, E.H. and M. Loss. 1997. Analysis. Providence, RI, American Mathematical Society.

Lü, H., D. O'Regan and R.P. Agarwal. 2005. Positive solutions for singular $p$-Laplacian equations with sign changing nonlinearities using inequality theory. Appl. Math. Comput., 165(3), 587-597.

Oerlemans, J. 1981. Some basic experiments with a verticallyintegrated ice sheet model. Tellus, 33(1), 1-11.

Paterson, W.S.B. 1994. The physics of glaciers. Third edition. Oxford, etc., Elsevier.

Picasso, M., J. Rappaz, A. Reist, M. Funk and H. Blatter. 2004. Numerical simulation of the motion of a two-dimensional glacier. Int. J. Num. Meth. Eng., 60(5), 995-1009.

Raviart, P.A. 1970. Sur la résolution de certaines équations paraboliques non linéaires. J. Funct. Anal., 5(2), 299-328.

Van den Berg, J., R.S.W. van de Wal and J. Oerlemans. 2006. Effects of spatial discretization in ice-sheet modelling using the shallowice approximation. J. Glaciol., 52(176), 89-98.

Van der Veen, C.J. 1999. Fundamentals of glacier dynamics. Rotterdam, A.A. Balkema.

Vialov, S.S. 1958. Regularities of glacial shields movement and the theory of plastic viscous flow. IASH Publ. 47 (Symposium at Chamonix, 1958 - Physics of the Movement of the Ice), 266-275.

Weertman, J. 1957. On the sliding of glaciers. J. Glaciol., 3(21), 33-38.

Weertman, J. 1961. Stability of ice-age ice sheets. J. Geophys. Res., 66(11), 3783-3792.

Weertman, J. 1964. The theory of glacier sliding. J. Glaciol., 5(39), 287-303.

\section{APPENDIX A: MATHEMATICAL PROOFS}

\section{Proof of theorem 1}

Using the definition of $J$, the Hölder inequality, the Poincaré inequality and $p>q$, we can show there exists $c_{1}>0$ and $c_{2}>0$ such that:

$J(\eta) \geq c_{1}\left(\int_{-1}^{1}\left|\eta^{\prime}\right|^{p} \mathrm{~d} x\right)-c_{2}\left(\int_{-1}^{1}\left|\eta^{\prime}\right|^{p} \mathrm{~d} x\right)^{q / p}+\alpha \int_{-1}^{1}|\eta| \mathrm{d} x$ for all $\eta \in K$. Since $p>q>1$, the last inequality implies there exists $c_{3}>0$ and $c_{4}>0$ such that:

$$
J(\eta) \geq c_{3}\left(\int_{-1}^{1}\left|\eta^{\prime}\right|^{p} \mathrm{~d} x\right)-c_{4}
$$

for all $\eta \in K$. Define

$$
\bar{m}=\inf \{J(\eta) ; \quad \eta \in K\} .
$$

On one side, $J(0)=0$, and on the other side, by Equation (A1), the functional $J$ is bounded below. Thus $\bar{m}$ is well defined. Let $\left\{\eta_{\nu}\right\} \subset K$ be a minimizing sequence for Equation (A2), i.e. $J\left(\eta_{\nu}\right) \longrightarrow \bar{m}$. There exists an integer, $N$, such that for all $\nu>N$, we have $\bar{m}+1 \geq J\left(\eta_{\nu}\right)$. Using Equation (A1) and weak compactness, we can extract a subsequence, still denoted $\left\{\eta_{\nu}\right\}$, and find $\bar{\eta} \in W^{1, p}(-1,1)$ so that $\eta_{\nu} \rightarrow \bar{\eta}$ in $W^{1, p}(-1,1)$, weakly. Since $K$ is strongly closed and convex, then $K$ is weakly closed (see Brezis, 1999). It follows that $\bar{\eta} \in K$. Since $p>1$, the function $\xi \mapsto \frac{1}{p}|\xi|^{p}$ is convex. Corollary 3.24 of Dacorogna (2008) states the weak lower continuity of any functional, $I(u)=$ $\int_{a}^{b} f\left(u, u^{\prime}\right) \mathrm{d} x$ if $f$ is continuous, $\xi \rightarrow f(u, \xi)$ is convex and if $f$ satisfies $f(u, \xi) \geq c|u|^{r}$, where $a, b, c \in \mathbb{R}, a<b$ and $r \geq 1$. As an application of this result, we obtain that $J$ is weakly lower semicontinuous. As a consequence,

$$
\bar{m}=\liminf _{\nu \rightarrow \infty} J\left(\eta_{\nu}\right) \geq J(\bar{\eta}) \geq \bar{m} .
$$

\section{Proof of lemma 1}

Owing to theorem 1, we can consider a minimizer, $\bar{\eta}$, of $J$ in $K$. Consider the even non-increasing rearrangement (or Schwarz symmetrization) $\eta^{*}$ of $\bar{\eta}$. (See Lieb and Loss, 1997 for the definition; and the introduction of Brock, 2000 for relevant explanations.) The function $\eta^{*}$ remains positive and equal to zero at -1 and 1 . Moreover, $\eta^{*}$ satisfies the property

$$
\eta^{*} \in L^{r}(-1,1), \quad \int_{-1}^{1}\left(\eta^{*}\right)^{r} \mathrm{~d} x=\int_{-1}^{1} \bar{\eta}^{r} \mathrm{~d} x, \quad \forall r \in[1, p]
$$

(Lieb and Loss, 1997, p.73) and the property

$$
\eta^{*} \in W^{1, p}(-1,1), \quad \int_{-1}^{1}\left|\left(\eta^{*}\right)^{\prime}\right|^{p} \mathrm{~d} x \leq \int_{-1}^{1}\left|\bar{\eta}^{\prime}\right|^{p} \mathrm{~d} x
$$

(Lieb and Loss, 1997, p.175). From Equations (A3) and (A4), we deduce that $J\left(\eta^{*}\right) \leq J(\bar{\eta})$.

\section{Proof of lemma 2}

Consider the even function $\eta(x)=\alpha^{\frac{1}{n}}(1-|x|)$, which is in $K$. Recall that $p=n+1>2$ and $q=\frac{n}{2(n+1)}+1 \in\left(\frac{5}{4}, \frac{3}{2}\right)$. We have

$$
\begin{aligned}
& J(\eta)=\int_{-1}^{1} \frac{\alpha^{p / n}}{p} \mathrm{~d} x-\int_{-1}^{1} \frac{\alpha^{1+\frac{q}{n}}}{q}(1-|x|)^{q} \mathrm{~d} x \\
& +\int_{-1}^{1} \alpha^{1+\frac{1}{n}}(1-|x|) \mathrm{d} x \\
& =\alpha^{1+\frac{1}{n}}\left(c_{1}-c_{2} \alpha^{\frac{1}{2(n+1)}}\right),
\end{aligned}
$$

where $c_{1}$ and $c_{2}$ are two positive constants. Clearly, $J\left(\eta^{*}\right) \leq J(\eta)<0$ if $\alpha$ is large enough.

\section{Proof of lemma 3}

It is easy to see that $\eta^{*}(0)>0$. Indeed, if $\eta^{*}(0)=0$, then $\eta^{*}$ would be equal to zero everywhere because $\eta^{*}$ is continuous, non-negative, non-increasing in $(0,1)$ and 
$\eta^{*}(1)=0$. As a consequence, $J\left(\eta^{*}\right)=0$ in contradiction to lemma 2. Define

$$
\gamma=\sup \left\{x \in(0,1) ; \quad \eta^{*}(x)>0\right\} \in(0,1] .
$$

We aim to show that $\gamma=1$. Assume that $\gamma<1$, and define $\zeta(x)=\gamma^{\frac{1-p}{p}} \eta^{*}(\gamma x) \in K$. We are going to show that $J(\zeta)<$ $J\left(\eta^{*}\right)$ which is a contradiction with $J\left(\eta^{*}\right)=\inf \{J(\eta) ; \quad \eta \in K\}$. Using the definition of $J$, the change of variable $y=\gamma x$ and the fact that $\eta^{*}=0$ outside $(-\gamma, \gamma)$, we obtain

$$
\begin{aligned}
J(\zeta) & =\frac{1}{p} \int_{-1}^{1}\left|\left(\eta^{*}\right)^{\prime}(y)\right|^{p} \mathrm{~d} y \\
& -\frac{\alpha}{q} \gamma^{q \frac{1-p}{p}-1} \int_{-1}^{1}\left(\eta^{*}\right)^{q}(y) \mathrm{d} y+\alpha \gamma^{\frac{1-p}{p}-1} \int_{-1}^{1} \eta^{*}(y) \mathrm{d} y .
\end{aligned}
$$

Using $\gamma^{q \frac{1-p}{p}-1}>\gamma^{\frac{1-p}{p}-1}>1$ and $J\left(\eta^{*}\right)<0$ (lemma 2), we obtain:

$$
\begin{aligned}
J(\zeta) & <\frac{1}{p} \int_{-1}^{1}\left|\left(\eta^{*}\right)^{\prime}(y)\right|^{p} \mathrm{~d} y \\
& +\gamma^{\frac{1-p}{p}-1}\left(-\frac{\alpha}{q} \int_{-1}^{1}\left(\eta^{*}\right)^{q}(y) \mathrm{d} y+\alpha \int_{-1}^{1} \eta^{*}(y) \mathrm{d} y\right) \\
& <\gamma^{\frac{1-p}{p}-1} J\left(\eta^{*}\right)<J\left(\eta^{*}\right) .
\end{aligned}
$$

\section{Proof of theorem 2}

Since $\eta^{*}$ is a minimizer of $J$ in $K$ then $\left.J\left[(1-\epsilon) \eta^{*}+\epsilon \xi\right)\right]=$ $J\left[\eta^{*}+\epsilon\left(\xi-\eta^{*}\right)\right] \geq J\left(\eta^{*}\right)$ for all $\varepsilon \in(0,1)$ and $\xi \in K$. It follows that

$$
\left\langle J^{\prime}\left(\eta^{*}\right), \xi-\eta^{*}\right\rangle=\lim _{\varepsilon \rightarrow 0^{+}} \frac{J\left[\eta^{*}+\varepsilon\left(\xi-\eta^{*}\right)\right]-J\left(\eta^{*}\right)}{\varepsilon} \geq 0,
$$

which becomes

$$
\begin{aligned}
\int_{-1}^{+1} & {\left[\left|\left(\eta^{*}\right)^{\prime}\right|^{p-2}\left(\eta^{*}\right)^{\prime}\left(\xi-\eta^{*}\right)^{\prime}\right.} \\
& \left.-\alpha\left(\eta^{*}\right)^{q-1}\left(\xi-\eta^{*}\right)+\alpha\left(\xi-\eta^{*}\right)\right] \mathrm{d} x \geq 0 .
\end{aligned}
$$

Fix $\varphi$ a smooth function with compact support. Then $\xi=$ $\eta^{*}+\tau \varphi$ belongs to $K$ if $|\tau|$ is sufficiently small because $\eta^{*}$ is continuous on $[-1,1]$ and $\eta^{*}>0$ on $(-1,1)$ by lemma 2 . Thus Equation (A5) implies:

$$
\tau \int_{-1}^{1}\left(\left|\left(\eta^{*}\right)^{\prime}\right|^{p-2}\left(\eta^{*}\right)^{\prime} \varphi^{\prime}-\alpha\left(\eta^{*}\right)^{q-1} \varphi+\alpha \varphi\right) \mathrm{d} x \geq 0 .
$$

This inequality is valid for all sufficiently small $\tau$, both positive and negative, and as a consequence

$$
\int_{-1}^{1}\left(\left|\left(\eta^{*}\right)^{\prime}\right|^{p-2}\left(\eta^{*}\right)^{\prime} \varphi^{\prime}-\alpha\left(\eta^{*}\right)^{q-1} \varphi+\alpha \varphi\right) \mathrm{d} x=0,
$$

for all smooth functions, $\varphi$, with compact support. By definition of the weak derivative, it follows that $\left|\left(\eta^{*}\right)^{\prime}\right|^{p-2}\left(\eta^{*}\right)^{\prime} \in$ $W^{1,1}(-1,1)$ and

$$
\left(\left|\left(\eta^{*}\right)^{\prime}\right|^{p-2}\left(\eta^{*}\right)^{\prime}\right)^{\prime}=-\alpha\left(\eta^{*}\right)^{q-1}+\alpha,
$$

almost everywhere on $(-1,1)$. Since the functions of $W^{1,1}(-1,1)$ are continuous on $[-1,1]$, then $\left(\eta^{*}\right)^{\prime}$ is also continuous and $\eta^{*}$ is continuously differentiable.

\section{Proof of lemma 4}

Similarly to lemma 2, define a two-parameter family $\eta_{\beta, \ell}(x)=\beta(1-|x| / \ell) \in \widehat{K}$, with $\eta_{\beta, \ell}(x)=0$ if $|x| \geq$ $\ell$. Recall $\widehat{\jmath}$ is defined in Equation (27). A straightforward integration shows

$$
\widehat{\jmath}\left(\eta_{\beta, \ell}\right)=\frac{2 \beta^{p}}{p \ell^{p-1}}+\alpha \beta \ell\left[1-\frac{2 \beta^{q-1}}{q(q+1)}\right] .
$$

Fix $\beta>\beta_{0}=[q(q+q) / 2]^{1 /(q-1)}$, which depends only on $n$, so that $1-2 \beta^{q-1} /[q(q+1)]<0$. From Equation (A7)

$$
\widehat{J}\left(\eta_{\beta, \ell}\right)=\frac{A}{\ell^{p-1}}-B \ell
$$

can be considered a function of $\ell$, with $A, B>0$. Choose a sequence $\ell_{k} \rightarrow \infty$, define $\eta_{k}=\eta_{\beta, \ell_{k}}$ and note that $\widehat{\jmath}\left(\eta_{k}\right) \rightarrow-\infty$ because $p>1$.

\section{APPENDIX B: THE STEADY-STATE CASE WITH SLIDING}

Consider Equation (6) in the steady case with mass balance determined by horizontal location, namely the following ODE for $H(x)$ :

$$
-\Gamma\left[(H+\bar{C}) H^{n+1}\left|H^{\prime}\right|^{n-1} H^{\prime}\right]^{\prime}=b(x) .
$$

Suppose the choice of constants as in Equation (7), assume $\bar{C}>0$, and use the mass-balance function, $b(x)$, given by Equation (30). As noted, it follows that the steady-state margin is at $L_{\mathrm{m}}=750 \mathrm{~km}$. The steady-state case we consider solves these conditions:

$$
\begin{aligned}
& H(x) \geq 0 \text { for all } x \text { in }(-L, L) \text {, and } \\
& \text { if } H(x)>0 \text { then } H \text { satisfies Equation (B1) at } x .
\end{aligned}
$$

This problem can be solved analytically up to a non-singular quadrature. We will convert the problem to a first-order ODE with a well-behaved right-hand side.

Because $\bar{C}>0$, however, a different scaling is appropriate relative to the choice in Equation (17) for a flat bed with no sliding. The correct scaling to de-singularize the margin is to replace $H$ with

$$
\omega=H^{\frac{2 n+1}{n}} .
$$

It is important to note that, unlike in the non-sliding flatbed case, the factor $(H+\bar{C})$ in the degenerate diffusivity, $D=(H+\bar{C}) H^{n+1}\left|H^{\prime}\right|^{n-1}$, has a nonzero limit at the margin. In the new variable $\omega$, Equation (B1) becomes

$$
-r^{n} \Gamma\left[\left(\omega^{r}+\bar{C}\right)\left|\omega^{\prime}\right|^{n-1} \omega^{\prime}\right]^{\prime}=b(x),
$$

where we have used the new exponent, $r=n /(2 n+1)$.

Symmetry implies that the flux at $x=0$ is zero. In terms of $\omega$, the flux is a constant multiple of $-\left(\omega^{r}+\bar{C}\right)\left|\omega^{\prime}\right|^{n-1} \omega^{\prime}$. Integrating Equation (B3) therefore gives

$$
-r^{n} \Gamma\left(\omega^{r}+\bar{C}\right)\left|\omega^{\prime}\right|^{n-1} \omega^{\prime}=Q(x),
$$

where $Q(x)$ is an odd $(Q(-x)=Q(x))$, continuous and piecewise-linear function satisfying

$$
Q(x)= \begin{cases}5 x, & \text { if } 0 \leq x \leq 500 \mathrm{~km} \\ 2500-10 x, & 500<x<750 \mathrm{~km} \\ 0, & 750 \mathrm{~km} \leq x\end{cases}
$$




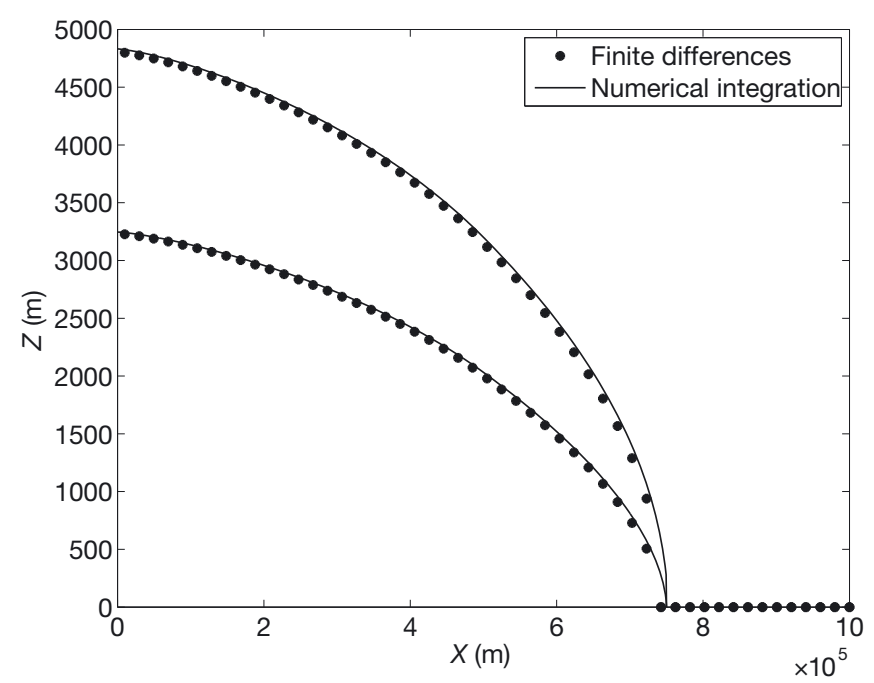

Fig. 8. Case 2: stationary solutions with sliding (lower curve) and without sliding (higher curve) (cf. Fig. 6). The solution is obtained by two different methods: finite difference method (dotted) and numerical quadrature of an ODE (solid).

Symmetry considerations imply $\omega^{\prime}<0$ for $x>0$, so the ice flow is in the positive $x$-direction for $x>0$. Equation (B4) can be written

$$
r^{n} \Gamma\left(\omega^{r}+\bar{C}\right)\left(-\omega^{\prime}\right)^{n}=Q(x),
$$

and the $n$th root computed:

$$
\omega^{\prime}=-\left[\frac{Q(x)}{r^{n} \Gamma\left(\omega^{r}+\bar{C}\right)}\right]^{1 / n} .
$$

The initial condition is $\omega\left(L_{\mathrm{m}}\right)=0$.

Equation (B7) is of the form $\omega^{\prime}=f(x, \omega)$. Because $\bar{C}>0$, the function $f$ is Lipschitz in the dependent variable $\omega$ : there is $\lambda$ independent of $x$ so that $\left|f\left(x, \omega_{2}\right)-f\left(x, \omega_{1}\right)\right| \leq \lambda\left|\omega_{2}-\omega_{1}\right|$. The derivative, $\partial f / \partial x$, has a singularity at $x=L_{\mathrm{m}}$, however. In fact, $Q(x) \sim\left(L_{\mathrm{m}}-x\right)$ as $x \rightarrow L_{\mathrm{m}}^{-}$so $F(x, \omega) \sim\left(L_{\mathrm{m}}-x\right)^{1 / n}$ as $x \rightarrow L_{\mathrm{m}}^{-}$. This feature makes numerical solution by any means less accurate, but can be fixed by a transformation:

$$
\xi=\left(L_{\mathrm{m}}-x\right)^{1 / n} .
$$

Let $\Omega(\xi)=\omega(x)$ be the new dependent variable. Then

$$
\Omega^{\prime}=n \xi^{n-1}\left[\frac{Q\left(L_{m}-\xi^{n}\right)}{q^{n} \Gamma\left(\Omega^{q}+\bar{C}\right)}\right]^{1 / n}
$$

The initial condition is $\Omega(\xi)=0$ and we seek the solution on the interval $0 \leq \xi \leq L_{\mathrm{m}}^{1 / n}$. Equation (B8) is Lipschitz in the dependent variable $\Omega$, and it is continuously differentiable in $\xi$ at $\xi=0$. This initial-value problem has a unique solution, and it is sufficiently regular to allow highly accurate numerical solution by an ODE solver. Figure 8 shows the solution produced by numerically integrating this initialvalue problem, and compares it to the finite-difference solution obtained by solving the time-dependent problem and converging to steady state. 